\title{
Evaluation of Droplet Digital PCR Assay for the Diagnosis of Candidemia in Blood Samples
}

\section{OPEN ACCESS}

Edited by:

Ying-Chun Xu,

Peking Union Medical College

Hospital (CAMS), China

Reviewed by:

Ott Scheler,

Tallinn University of Technology,

Estonia

Weida Liu,

Chinese Academy of Medical

Sciences and Peking Union Medical

College, China

*Correspondence:

Dongmei Shi

shidongmei28@163.com

Xiaojie Zhang

qlzxj@126.com

${ }^{\dagger}$ These authors share first authorship

Specialty section: This article was submitted to Antimicrobials, Resistance and Chemotherapy,

a section of the journal

Frontiers in Microbiology

Received: 25 April 2021

Accepted: 26 July 2021 Published: 03 September 2021

Citation:

Chen B, Xie Y, Zhang N, Li W, LiU C, Li D, Bian S, Jiang Y, Yang Z, Li R, Feng $Y$, Zhang $X$ and Shi D (2021)

Evaluation of Droplet

Digital PCR Assay for the Diagnosis of Candidemia in Blood Samples.

Front. Microbiol. 12:700008.

doi: 10.3389/fmicb.2021.700008

\begin{abstract}
Biao Chen ${ }^{1,2+}$, Yingguang Xie ${ }^{3 \dagger}$, Ning Zhang ${ }^{1}$, Wenqiang $\mathrm{Li}^{3}$, Chen Liu', Dongmei $\mathrm{Li}^{4}$, Shaodong Bian', Yufeng Jiang ${ }^{2,5}$, Zhiya Yang ${ }^{1}$, Renzhe Li ${ }^{5}$, Yahui Feng ${ }^{6}, X_{i a o j i e}$ Zhang $^{2 *}$ and Dongmei Shi ${ }^{1,7 *}$

'The Laboratory of Medical Mycology, Jining No. 1 People's Hospital, Jining, China, ${ }^{2}$ Postdoctoral Mobile Station of Shandong University of Traditional Chinese Medicine, Jinan, China, 'Intensive Care Unit, Jining No. 1 People's Hospital, Jining, China, ${ }^{4}$ Department of Microbiology and Immunology, Georgetown University Medical Center, Washington, DC, United States, ${ }^{5}$ Clinical Laboratory, Jining No. 1 People's Hospital, Jining, China, ${ }^{6}$ Clinical Medicine College, Jining Medical College, Jining, China, 'Department of Dermatology, Jining No. 1 People's Hospital, Jining, China
\end{abstract}

Numerous studies have shown that droplet digital PCR (ddPCR) is a promising tool for the diagnosis of pathogens, especially in samples with low concentrations of pathogenic DNA. An early diagnosis of candidemia is critical for the effective treatment of patients. In this study, we evaluated the sensitivity and specificity of ddPCR assay for Candida DNA detection both in vitro by mixing fungal cells with human blood and in vivo by analyzing blood samples from infected mice and patients with suspected candidemia. The results showed that ddPCR assay could detect a minimum of 4.5 DNA copies per reaction in blood samples. ddPCR showed higher sensitivity and specificity for Candida DNA detection than traditional culture and quantitative PCR (qPCR) methods and also exhibited significantly better positive and negative predictive values than the culture and GPCR methods that were commonly used in clinical practice. Hence, our study demonstrates that ddPCR assay is a promising method for the timely diagnosis of candidemia and could be useful for monitoring the treatment of candidemia.

Keywords: droplet digital PCR, diagnosis, candidemia, blood samples, sensitivity, specificity

\section{INTRODUCTION}

Candidemia is a leading cause of fungal infections among neonates and infants, and it also affects immunocompromised adults (Golan et al., 2005; Mantadakis et al., 2018). The incidence of candidemia is 13.3 per 100,000 people, and the mortality ranges from 36 to $50 \%$ (Ngamchokwathana et al., 2021). Given the rapid and fatal course of candidemia, timely and effective treatment depends on rapid and accurate diagnosis of this invasive fungal infection (Pfaller and Diekema, 2007; Schroeder et al., 2020).

The traditional procedure for diagnosing candidemia relies on blood cultures for the isolation of Candida spp. However, this method has low sensitivity and requires large volumes of blood (Clancy and Nguyen, 2013; Pappas et al., 2018). The growth, isolation, and identification of Candida spp. routinely take $24-48 \mathrm{~h}$. This time-consuming approach takes even longer time when dealing with slower-growing fungal species, which will prevent the timely diagnosis and 
the initiation of appropriate anti-fungal treatment. The first $12-48 \mathrm{~h}$ post-infection is a critical period since delaying treatment significantly increases the mortality of candidemia (Tumbarello et al., 2007; Tulasidas et al., 2018). Hence, the broad-spectrum empirical anti-fungal treatment is frequently used for high-risk patients. However, this will unnecessarily increase costs and risk of adverse reactions in these patients (Tumbarello et al., 2007; de Pauw et al., 2008).

Recent efforts in the development of early diagnostic methods have focused on the identification of molecular markers of pathogens. Also, quantitative PCR (qPCR)-based methods for DNA detection have become more popular than other non-culture methods. They facilitate the rapid diagnosis of bloodstream fungal infections, allowing for the initiation of species-oriented therapy as soon as $6 \mathrm{~h}$ after the onset of sepsis (Mcmullan et al., 2008). However, the sensitivity, accuracy, and replicability of these techniques do not fulfill the requirements of clinical practice, especially in the cases of samples with low abundance of pathogen DNA or with insufficient volumes of blood, as is common in premature or young infants (Nyaruaba et al., 2019).

Droplet digital PCR (ddPCR), based on water-oil emulsion droplet technology, is a new PCR method for nucleic acid detection that allows more accurate quantification of DNA templates (Schell et al., 2012). Each sample is partitioned into approximately 20,000 droplets before being subjected to the procedure. Ideally, each droplet contains one target molecule or none. Unlike qPCR, there is no need to establish a standard curve. The number of droplets with their amplification products is counted at the end of amplification, allowing for an estimate of template concentration based on Poisson's Law of Small Numbers. With a high sensitivity to detect low copies of DNA, ddPCR assay has been applied in several viral infections such as human papilloma virus (Schiavetto et al., 2021), hepatitis $B$ virus (Lillsunde), chromosomally integrated human herpes virus 6 (Sedlak et al., 2014), and Mycobacterium spp. detection for tuberculosis (Yang et al., 2017) and leprosy (Cheng et al., 2019). However, the clinical utility of ddPCR assay for fungal detection remains unclear. To further optimize the ddPCR assay for the detection of Candida spp., the diagnostic performance of ddPCR assay was compared with conventional culture and qPCR assays using blood samples from mice with experimental candidemia and patients with suspected candidemia. The specificity of ddPCR for Candida spp. was also estimated using non-Candida fungal templates in vitro.

\section{MATERIALS AND METHODS}

\section{Fungal and Bacterial Strains and Culture Conditions}

Several typical fungal and bacterial strains, including Candida albicans (SC 5314), C. tropicalis (CBS 8072), C. parapsilosis (ATCC 22019), C. krusei (CBS 6451), Trichophyton rubrum (ATCC 4438), Aspergillus fumigatus (MAY 3626), Staphylococcus aureus (ATCC 25923), Escherichia coli (ATCC 35218), Pseudomonas aeruginosa (ATCC 27853), and Streptococcus pneumoniae (ATCC 49619), were obtained from the Laboratory of Medical Mycology, Jining No. 1 People's Hospital, Shandong, China. Fungal cells were inoculated on Sabouraud-glucose-agar (SDA) plates for $72 \mathrm{~h}$ at $30^{\circ} \mathrm{C}$, and bacterial strains were cultured on blood agar plates (Jinan Baibo Biotechnology Co., Ltd.) for $24 \mathrm{~h}$ at $37^{\circ} \mathrm{C}$.

\section{DNA Extraction}

Fungal DNA extraction was performed according to the manufacturer's (OMEGA) instructions. Each blood sample $(0.25 \mathrm{ml})$ was transferred into sterile micro-centrifuge tubes containing $25 \mu \mathrm{l} \mathrm{OB}$ protease solution and $250 \mu \mathrm{l} \mathrm{BL}$ buffer. After incubation at $65^{\circ} \mathrm{C}$ for $10 \mathrm{~min}, 260 \mu \mathrm{l}$ ethanol was added to each blood sample, which was then vortexed at the maximum speed for $20 \mathrm{~s}$ and transferred into HiBind ${ }^{\circledR}$ DNA mini-columns and centrifuged at $10,000 \mathrm{~g}$ for $1 \mathrm{~min}$. In a new collection tube, $500 \mu \mathrm{l} \mathrm{HBC}$ buffer was added to a DNA mini-column. After centrifuging again at $1,000 \mathrm{~g}$ for $1 \mathrm{~min}$, DNA was washed with $700 \mu \mathrm{l}$ wash buffer and finally collected in a nuclease-free $2-\mathrm{ml}$ micro-centrifuge tube with $200 \mu$ l elution buffer at $65^{\circ} \mathrm{C}$ at $13,000 \mathrm{~g}$ for $1 \mathrm{~min}$. The DNA samples were immediately stored at $-20^{\circ} \mathrm{C}$ until use. All laboratory equipment was disinfected and decontaminated using UV-treatment prior to DNA preparation. Laboratory equipment and surfaces were also regularly disinfected using $10 \%$ bleach and $70 \%$ ethanol before conducting any analysis.

\section{Quantitative PCR}

Candida albicans in blood samples was detected by a qPCR assay using the primers (forward: 5'-TCAAAACTT TCAACAACGGATCTC-3'; and reverse: 5'-CGCATTTCGCTG CGTTCT-3') synthesized by China Electronics Huada Technology Co. Ltd. In a total volume of $25 \mu \mathrm{l}$ qCR reaction, $12.5 \mu \mathrm{l}$ TB Green Premix Ex Taq II was mixed with $4.84 \mu \mathrm{l}$ of DNA (66 ng), $1 \mu \mathrm{l}(25 \mathrm{pmol})$ each of forward primer and reverse primer, and $5.66 \mu \mathrm{l} \mathrm{H}_{2} \mathrm{O}$. The amplification was performed under the following conditions: the initial denaturation at $95^{\circ} \mathrm{C}$ for $30 \mathrm{~s}$ and 40 cycles of amplification at $95^{\circ} \mathrm{C}$ for $5 \mathrm{~s}$ and $57^{\circ} \mathrm{C}$ for $30 \mathrm{~s}$. Bio-Rad CFX Maestro detection system was used for amplification, detection, and data analysis. Internal, positive, and negative controls were included in each experiment. To avoid the risk of false positive results due to laboratory contamination, all the experimental setups were performed in a biosafety cabinet.

\section{Droplet Digital PCR}

All ddPCR assays were conducted using QX200 Droplet Digital PCR system (Bio-Rad, United States) in a $22-\mu$ l volume system. The primers and probe for C. albicans were as follows: forward primer 5'-TCAAAACTTTCAACAACGGATCTC-3'; reverse primer 5'-CGCATTTCGCTGCGTTCT-3', and the probe: 5'-TGGTTCTCGCATCGAT-3'. The probe was labeled with FAM. The probe and two primers were synthesized by China Electronics Huada Technology Co. Ltd. Internal, positive, and negative controls were included in each ddPCR run. Each reaction contained $11 \mu \mathrm{l}$ Bio-Rad $2 \times$ ddPCR Supermix, $1.1 \mu \mathrm{l}$ forward primer $(18 \mu \mathrm{M})$ and $1.1 \mu \mathrm{l}$ reverse primer $(18 \mu \mathrm{M})$, 
$1.1 \mu \mathrm{l}$ probe $(5 \mu \mathrm{M}), 4.84 \mu \mathrm{l}$ template DNA (66ng), and $2.86 \mu \mathrm{l}$ $\mathrm{H}_{2} \mathrm{O}$. The PCR mix and sample DNA were thoroughly mixed before loading to a droplet generator cartridge. After droplet generation oil was added, the DNA mixtures were placed into a droplet generator (Bio-Rad, United States). Droplet-partitioned samples were transferred to a ddPCR 96-well plate, which was then sealed at $180^{\circ} \mathrm{C}$ using a PX1TM PCR plate sealer (BioRad) before being amplified in a thermal cycler. The PCR annealing temperature was optimized at $57^{\circ} \mathrm{C}$, as this temperature provided a clear separation between DNA-positive and negative droplets in the preliminary analysis. PCR was performed in a T100TM thermal cycler under the following conditions: $95^{\circ} \mathrm{C}$ for $10 \mathrm{~min}$, followed by 40 cycles of $94^{\circ} \mathrm{C}$ for $30 \mathrm{~s}$ and $57^{\circ} \mathrm{C}$ for $60 \mathrm{~s}$, and the final extension at $98^{\circ} \mathrm{C}$ for $10 \mathrm{~min}$. Moreover, a ramp rate was set up at $2^{\circ} \mathrm{C} / \mathrm{s}$ in the PCR program for every amplification step. After amplification, droplets remained at $4^{\circ} \mathrm{C}$ for at least $30 \mathrm{~s}$. DNA targets in each droplet were quantized by thermal cycling and analyzed by the Bio-Rad QX200TM droplet reader. To limit laboratory contamination, all the setup procedures before thermal cycler use were performed in a biosafety cabinet.

\section{Estimation of the Limit of Detection and Specificity}

To calculate the limit of detection (LOD) of C. albicans DNA in blood samples, 10 -fold dilutions of $1 \times 10^{-1} \mathrm{ng} / \mu \mathrm{l}$ stock of C. albicans DNA were prepared with PBS. The final range of DNA dilution was $10^{-1}-10^{-7}$ from the C. albicans stock concentration $(1 \mathrm{ng} / \mu \mathrm{l})$ in ddPCR analysis. The specificity of ddPCR for C. albicans DNA detection was evaluated with the DNA extractions from C. tropicalis, C. parapsilosis, C. krusei, Staphylococcus aureus, Escherichia coli, Pseudomonas aeruginosa, Streptococcus pneumoniae, and Klebsiella pneumoniae using the same primer set for C. albicans assay. DNA stock used in this experiment was quantified using NanoDrop One/OneC (Thermo, USA).

\section{Experimental Candidemia in Mice With C. albicans}

Experimental candidemia was established in mice by intravenous challenge with cultured cells of $C$. albicans. In each experiment, $\mathrm{BALB} / \mathrm{c}$ female mice (aged 4-8 weeks) were infected by the tail vein injection of $C$. albicans cells in $500 \mu \mathrm{l}$ sterile PBS at $2 \times 10^{6} \mathrm{CFU}, 2 \times 10^{5} \mathrm{CFU}$, or $2 \times 10^{4} \mathrm{CFU}$, respectively. Each concentration of $C$. albicans was injected into 15 female mice. Animals were randomly assigned to different groups. At subsequent time points (days 1,3 , and 7 post-infection), the mice were euthanized by carbon dioxide. The blood samples were obtained by orbital puncture, collected in EDTA tubes, and divided into three parts for quantitative culturing on SDA agar plates, conventional qPCR and ddPCR testing.

\section{Blood Sampling From Patients With Suspected Candidemia}

Forty-five blood samples of hospitalized patients with suspected candidemia were collected between January 2019 and April 2020 at Jining No. 1 People's Hospital (Jining, China). The blood samples were collected into EDTA tubes for immediate detection or storage at $-80^{\circ} \mathrm{C}$ for further use. All samples were ultimately used for culture, and the extracted DNA samples were used for qPCR and ddPCR analyses.

\section{Ethics Statement}

The study was approved by Jining Medical College and Jining No. 1 People's Hospital, Shandong, China (Approval No. 2020028). The guidelines given by the Genetic Risk Prediction Studies were followed. Written informed consent was obtained from each participant or from legal guardians of any minors. Meanwhile, all the animal experiments were conducted as per the guidelines of the Animal Ethics Committee of Jining No. 1 People's Hospital. The animal study was reviewed and approved by the Jining Medical College, Shandong.

\section{Statistical Analysis}

We considered the qPCR or culture assay as "gold standard" for fungal identification (Momin et al., 2020). Then, we used these techniques to estimate positive detection rates, sensitivities, specificities, and positive predictive and negative predictive values of ddPCR in suspected candidemia. IBM SPSS Statistics for Windows, Version 24.0 (IBM Corp., Armonk, NY) was used for all statistical analyses.

\section{RESULTS}

\section{Designed ddPCR Assay Is Specific to C. albicans}

The primers and probe designed in this study for both qPCR and ddPCR were genus-specific for C. albicans. To analyze the general specificity of the ddPCR method, we used the same primers, probe, and amplification procedure against genomic DNA preparations from other fungal and bacterial species. These other pathogens are also commonly found in bacteremia or candidemia blood samples. The ddPCR results showed that C. tropicalis, C. parapsilosis, and C. krusei could be amplified; however, all the bacterial samples were negative with C. albicansspecific primers, even though the concentrations of DNA template were $66 \mathrm{ng}$ per ddPCR reaction. Meanwhile, the negative controls showed no amplified product. Since all the bacterial samples with non-target DNA were negative, ddPCR assay showed good specificity in detecting Candida spp. (Figure 1).

\section{ddPCR Has Improved Quantitative Range in Comparison to qPCR}

The minimum concentration necessary for DNA in the blood samples for ddPCR detection was first determined in vitro by 10 -fold serial dilution from an initial concentration of $1 \times 10^{-1} \mathrm{ng} /$ $\mu \mathrm{L}$ DNA from C. albicans strain (SC 5314). The DNA copies per reaction ranged from 142,600 to 0 . The fraction of positive droplets ranged from 0.9977 to 0 . The target copies/droplet ranged from 15.8058 to 0 . And, the results showed that the LOD for ddPCR detection for C. albicans was 4.5 copies per 


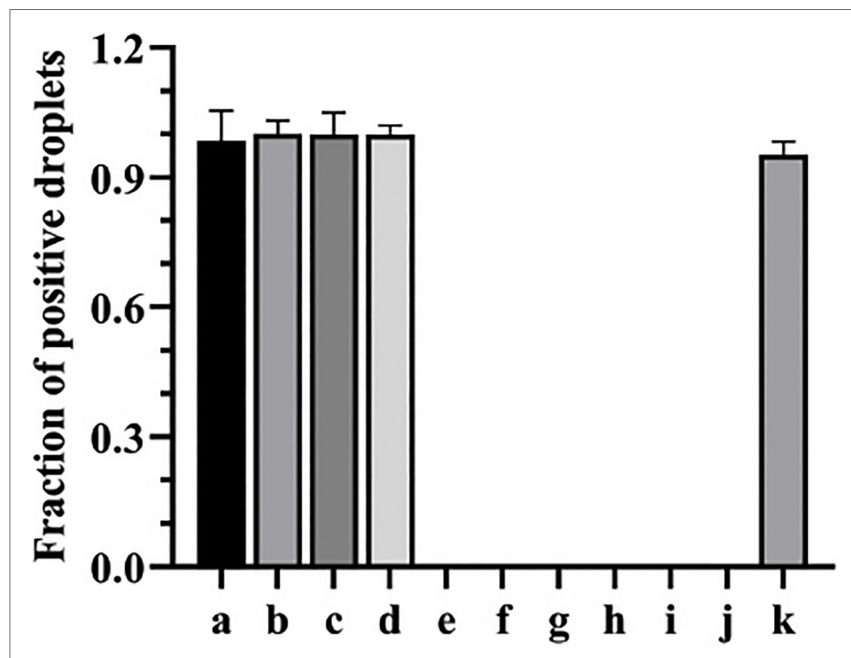

FIGURE 1 | The specificity of ddPCR assay for the detection of Candida albicans. a, b, c, d, e, f, g, h, i, and g represent the fraction of positive droplets of Candida tropicalis, C. parapsilosis, C. krusei, C. albicans, Trichophyton rubrum, Aspergillus fumigatus, Staphylococcus aureus, Escherichia coli, Pseudomonas aeruginosa, and Streptococcus pneumoniae, respectively. $\mathrm{k}$ represents positive control (C. albicans). reaction, provided by $2 \times 10^{-7}$-fold dilution sample. When compared with ddPCR, the minimum number of DNA concentration for qPCR must be greater than the number of DNA concentration in $1 \times 10^{-6}$-fold dilution sample, since the qPCR method requires a $34.3 \mathrm{Cq}$ value ( $1 / \mathrm{Cq}$ value with 0.029$)$ for $C$. albicans detection at the $1 \times 10^{-6}$-fold dilution point. This Cq value was almost indistinguishable from the $36.7 \mathrm{Cq}$ value $(1 / \mathrm{Cq}$ value with 0.027$)$ obtained from the negative control (Figure 2 and Supplementary Table 1). Therefore, the results indicated that the ddPCR method had at least 5-fold higher sensitivity than the qPCR method for C. albicans DNA detection in blood samples.

\section{ddPCR Detected Candidemia in vivo}

To further evaluate the performance of ddPCR in the detection of $C$. albicans infection in vivo, we used infected mice to mimic candidemia in humans. Blood samples from infected mice were collected at day 1,3 , and 7 post-infection with different concentrations of $C$. albicans cells. As expected, the detection capacity of fraction of positive droplets by ddPCR method increased with higher dosages of C. albicans. For example, at day 1 , the fraction of positive droplets were found to be $0.00055,0.00114$, and 0.00085 for inoculations of $1 \times 10^{4} \mathrm{CFU}, 1 \times 10^{5} \mathrm{CFU}$, and $1 \times 10^{6} \mathrm{CFU}$, respectively (Figure 3). The fractions of positive droplets were 0.00161, 0.00531 , and 0.01584 at day 3 , and then dropped slightly to $0.00146,0.00132$, and 0.00488 at day 7 post-infection. Indeed, this peak at day 3 was consistent with the peak infection course in the mouse infection model. These mice often died or became seriously ill during the 3-5 days postinfection. These results suggested that the ddPCR assay was not only useful for an early diagnosis but also provided prognostic value for candidemia management.

\section{ddPCR Showed Higher Sensitivity in Detecting Candida Among Suspected Candidemia Samples}

A total of 45 blood samples from hospitalized patients with suspected candidemia were used to further evaluate the effectiveness of ddPCR in detecting Candida in clinical samples. The detection rates of the ddPCR method were also compared with those obtained from culture and qPCR methods. The results showed that the positive detection rates were $44 \%$ for culture method $(n=20), 51 \%$ for qPCR $(n=23)$, and $73 \%$ for ddPCR $(n=32)$, respectively, of which 29 patients $(64 \%)$ were positive either by culture or qPCR method alone, or by both methods, which was inferior to the positive rate of the ddPCR method. When compared with the two other methods, the sensitivity of ddPCR was much higher, with 94 vs. $69 \%$ for culture and $79 \%$ for qPCR method (Table 1). With $91 \%$ positive predictive value and $85 \%$ negative predictive value, the ddPCR method was more sensitive and efficient than either culture or $\mathrm{qPCR}$ method for candidemia diagnosis.

\section{DISCUSSION}

This study demonstrated that ddPCR assay facilitated accurate quantification of Candida DNA in human blood. As no standard curve is needed, ddPCR also allows for the direct comparison of Candida concentrations measured by different laboratories. Previous studies found that variation in quantification between technical replicates was considerably lower for ddPCR than for qPCR (Sedlak et al., 2014; Wu et al., 2018). Hence, ddPCR has advantages over other qPCR methods for pathogen DNA detection in clinical samples. Despite the popularity of qPCR, differences between testing instruments, different agents or suppliers, or variations in the standard curves can confound the interpretation and the portability of results (Chindamporn et al., 2018; Cutarelli et al., 2021).

The ddPCR method showed good reproducibility and high specificity in this study. As expected, the sensitivity of ddPCR assay for Candida spp. detection in blood samples was significantly higher than that of culture or qPCR methods. The sensitivity of ddPCR (94\%) was higher than the $86 \%$ observed in neonates in a previous study (Li et al., 2019). Given that our patients were mainly adults, this difference could be due to quantitative differences of fungal cells in the blood between adults and neonates after the onset of disease (sampling time). The ddPCR method also has other diagnostic advantages over conventional qPCR, including lower sample volume requirements and faster execution times, because the thermocycling times are shorter (De falco et al., 2021).

The specificity of the probe sets used in ddPCR assays for specific microbes is often established using closely related species and unrelated species such as human DNA. The probe in the present study was $100 \%$ specific for Candida genus and did not hybridize with human DNA. The probe was designed based on two observations. First, about $80 \%$ of current systemic fungal infections are caused by Candida species; second, 


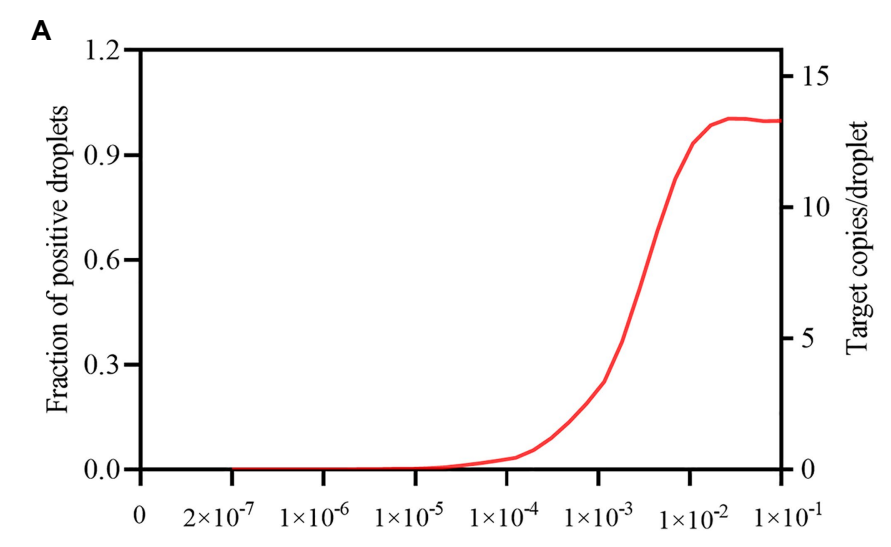

DNA concentrations $(\mathrm{ng} / \mathrm{mL})$

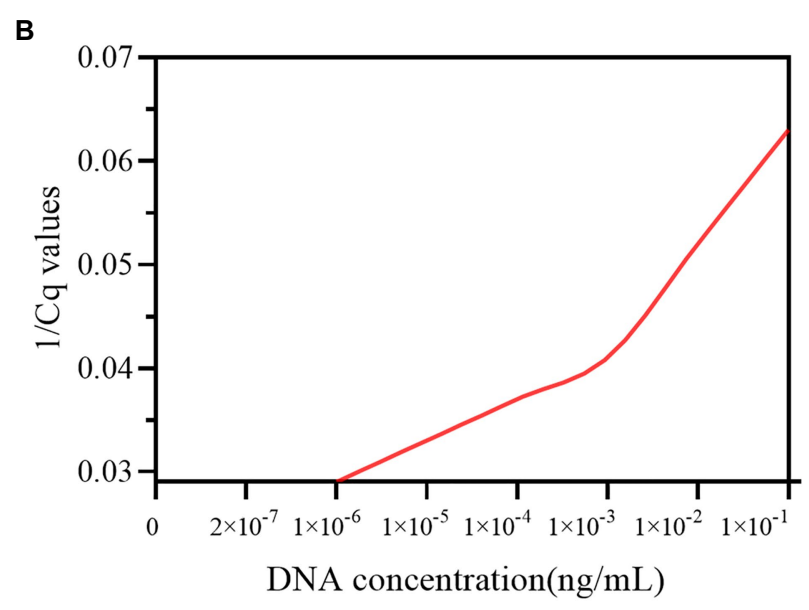

FIGURE 2 | The sensitivity of the ddPCR and qPCR assays in detecting candidemia. (A) Shows the sensitivity of ddPCR in detecting candidemia. X-axis represents the theoretical dynamic range of DNA concentration $\left(2 \times 10^{-7}-1 \times 10^{-5} \mathrm{ng} / \mathrm{ml}\right)$. Y-axis on the left represents the fraction of positive droplets detected by ddPCR. Y-axis on the right represents target copies/droplet detected by ddPCR. (B) Shows the sensitivity of qPCR in detecting candidemia. $\mathrm{X}$-axis indicates the DNA concentration, ranging from $2 \times 10^{-7}-1 \times 10^{-1} \mathrm{ng} / \mathrm{ml}$ Y-axis indicates the $1 / \mathrm{Cq}$ value detected by $\mathrm{qPCR}$.

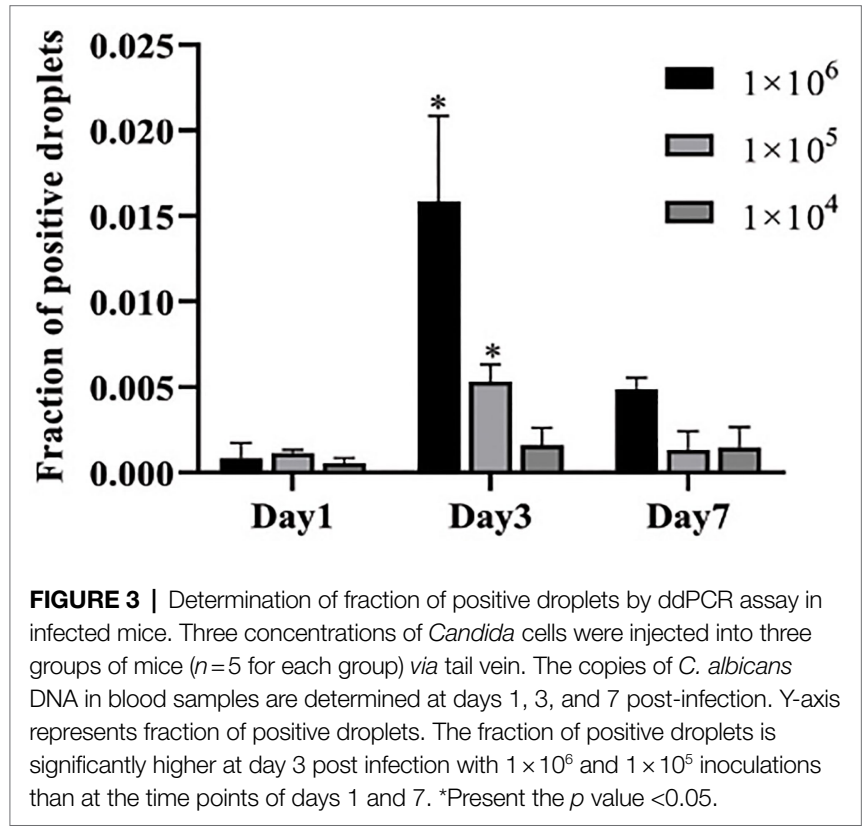

approximately $60 \%$ of fungal isolates are C. albicans (Kim et al., 2020). However, non-albicans species, such as $C$. tropicalis, C. parapsilosis, C. glabrata, and C. krusei, are also prevalent (Krcmery and Barnes, 2002; Wisplinghoff et al., 2014). The probe and primer set used in this study could detect DNA from non-albicans species, such as C. tropicalis, $C$. parapsilosis and C. glabrata, which would reduce the likelihood of overlooking the presence of non-albicans species in clinical practice. Meanwhile, this probe and primer set based on the Candida genus could not detect other fungal pathogens, such as Aspergillus spp. and T. rubrum, and bacteria such as $S$. aureus, E. coli, P. aeruginosa, and S. pneumoniae, which are commonly found in patients with bacteremia.
TABLE 1 | Positive and negative predictive values, sensitivity and specificity of different diagnostic assays in patients with suspected candidemia $(n=45$, including two neonates).

\begin{tabular}{lccccc}
\hline $\begin{array}{l}\text { Diagnostic } \\
\text { method }\end{array}$ & $\begin{array}{c}\text { Detection } \\
\text { rate (\%) }\end{array}$ & $\begin{array}{c}\text { Sensitivity } \\
\text { (\%) }\end{array}$ & $\begin{array}{c}\text { Specificity } \\
\text { (\%) }\end{array}$ & $\begin{array}{c}\text { Positive } \\
\text { predictive } \\
\text { value (\%) }\end{array}$ & $\begin{array}{c}\text { Negative } \\
\text { predictive } \\
\text { value (\%) }\end{array}$ \\
\hline Culture & 44 & 69 & 100 & 100 & 64 \\
RT-PCR & 64 & 89 & 76 & 86 & 81 \\
ddPCR & 73 & 94 & 79 & 91 & 85
\end{tabular}

RT-PCR, Real Time-PCR; and ddPCR, droplet digital PCR.

Both high sensitivity and specificity in ddPCR could facilitate the studies on other fungal detections in future. For example, an assay to identify fungal species in a single run of ddPCR assay could be developed using two probes, of which one probe could be based on the genus and the second probe could be based on the particular species. We will continue to evaluate the sensitivity and specificity of ddPCR in larger candidemia cohorts, and in other types of clinical samples (e.g., urine or BAL). Given its higher sensitivity, a prospective clinical study using the ddPCR assay for the identification of patients at high risk for invasive fungal infections is underway.

The high specificity and sensitivity of ddPCR facilitates its application in the early diagnosis of candidemia. In this study, two blood samples were obtained from infants suspected with candidemia. Both infants were detected positive by ddPCR assay but negative by culture and qPCR assays. Following the diagnosis of candidemia based on the ddPCR assay, these two infant patients were immediately given antifungal treatment and quickly cured. In order to avoid false negative result, a series of diluted samples need to be prepared and detected by ddPCR assay in the early diagnosis of candidemia. 
DATA AVAILABILITY STATEMENT

The raw data supporting the conclusions of this article will be made available by the authors, without undue reservation.

\section{ETHICS STATEMENT}

The studies involving human participants were reviewed and approved by the Jining Medical College and Jining No. 1 People's Hospital, Shandong, China (2020-028). Written informed consent to participate in this study was provided by the participants' legal guardian/next of kin. The animal study was reviewed and approved by the Jining Medical College, Shandong, China (2020-028).

\section{AUTHOR CONTRIBUTIONS}

BC was mainly for investigation, formal analysis, and writingoriginal draft. NZ was mainly for investigation and formal analysis. WL, DL, CL, SB, YJ, ZY, RL, and YF were mainly for collecting samples. YX, XZ, and DS were mainly for designing the study, funding acquisition, investigation, and review and

\section{REFERENCES}

Cheng, X., Sun, L., Zhao, Q., Mi, Z., Yu, G., Wang, Z., et al. (2019). Development and evaluation of a droplet digital PCR assay for the diagnosis of paucibacillary leprosy in skin biopsy specimens. PLoS Negl. Trop. Dis. 13:e0007284. doi: 10.1371/journal.pntd.0007284

Chindamporn, A., Chakrabarti, A., Li, R., Sun, P. L., Tan, B. H., Chua, M., et al. (2018). Survey of laboratory practices for diagnosis of fungal infection in seven Asian countries: An Asia fungal working group (AFWG) initiative. Med. Mycol. 56, 416-425. doi: 10.1093/mmy/myx066

Clancy, C. J., and Nguyen, M. H. (2013). Finding the "missing 50\%" of invasive candidiasis: how nonculture diagnostics will improve understanding of disease spectrum and transform patient care. Clin. Infect. Dis. 56, 1284-1292. doi: $10.1093 / \mathrm{cid} / \mathrm{cit} 006$

Cutarelli, A., De Falco, F., Uleri, V., Buonavoglia, C., and Roperto, S. (2021). The diagnostic value of the droplet digital PCR for the detection of bovine deltapapillomavirus in goats by liquid biopsy. Transbound. Emerg. Dis. doi: 10.1111/tbed.13971 [Epub ahead of print].

De Falco, F., Corrado, F., Cutarelli, A., Leonardi, L., and Roperto, S. (2021). Digital droplet PCR for the detection and quantification of circulating bovine deltapapillomavirus. Transbound. Emerg. Dis. 68, 1345-1352. doi: 10.1111/ tbed.13795

de Pauw, B., Walsh, T. J., Donnelly, J. P., Stevens, D. A., Edwards, J. E., Calandra, T., et al. (2008). Revised definitions of invasive fungal disease from the European Organization for Research and Treatment of cancer/ invasive fungal infections cooperative group and the National Institute of Allergy and Infectious Diseases mycoses study group (EORTC/MSG) consensus group. Clin. Infect. Dis. 46, 1813-1821. doi: 10.1086/588660

Golan, Y., Wolf, M. P., Pauker, S. G., Wong, J. B., and Hadley, S. (2005). Empirical anti-Candida therapy among selected patients in the intensive care unit: a cost-effectiveness analysis. Ann. Intern. Med. 143, 857-869. doi: 10.7326/0003-4819-143-12-200512200-00004

Momin, K. M., Milton, A. A. P., Ghatak, S., Thomas, S. C., Priya, G. B., Das, S., et al. (2020). Development of a novel and rapid polymerase spiral reaction (PSR) assay to detected Salmonella in pork and pork products. Mol. Cell. Probes. 50:101510. doi: 10.1016/j.mcp.2020.101510

Kim, E. J., Lee, E., Kwak, Y. G., Yoo, H. M., Choi, J. Y., Kim, S. R., et al. (2020). Trends in the epidemiology of candidemia in intensive care units editing. All authors contributed to the article and approved the submitted version.

\section{FUNDING}

This work was supported in part by grants from the National Natural Science Foundation of China (NM 81773337), the Key Research and Development Plan of Shandong Province (NM 2019GSF108191), the Key Research and Development Plan of Jining (NM2019SMNS008), the Doctoral Fund of Jining No.1 People's Hospital (2019004), the Technology Development Program of Shandong Province (2018WS469 and 2018WS477), the China Postdoctoral Science Foundation (2020T130073ZX), and the Natural Science Foundation of Shandong Province (NM ZR2020QH272), China.

\section{SUPPLEMENTARY MATERIAL}

The Supplementary Material for this article can be found online at: https://www.frontiersin.org/articles/10.3389/fmicb.2021.700008/ full\#supplementary-material

from 2006 to 2017: results from the Korean National Healthcare-Associated Infections Surveillance System. Front. Med. 7:606976. doi: 10.3389/ fmed.2020.606976

Krcmery, V., and Barnes, A. J. (2002). Non-albicans Candida spp. causing fungaemia: pathogenicity and antifungal resistance. J. Hosp. Infect. 50, 243-260. doi: $10.1053 /$ jhin.2001.1151

Li, H. T., Lin, B. C., Huang, Z. F., Yang, C.-Z., and Huang, W.-M. (2019). Clinical value of droplet digital PCR in rapid diagnosis of invasive fungal infection in neonates. Zhongguo Dang Dai ErKe Za Zhi 21, 45-51. doi: 10.7499/j.issn.1008-8830.2019.01.009

Mantadakis, E., Pana, Z. D., and Zaoutis, T. (2018). Candidemia in children: epidemiology, prevention and management. Mycoses 61, 614-622. doi: 10.1111/ myc. 12792

McMullan, R., Metwally, L., Coyle, P. V., Hedderwick, S., McCloskey, B., O’Neill, H. J., et al. (2008). A prospective clinical trial of a real-time polymerase chain reaction assay for the diagnosis of candidemia in nonneutropenic, critically ill adults. Clin. Infect. Dis. 46, 890-896. doi: 10.1086/528690

Ngamchokwathana, C., Chongtrakool, P., Waesamaae, A., and Chayakulkeeree, M. (2021). Risk factors and outcomes of non-albicans candida bloodstream infection in patients with candidemia at siriraj hospital-thailand largest national tertiary referral hospital. J. Fungi. 7:269. doi: 10.3390/jof7040269

Nyaruaba, R., Mwaliko, C., Kering, K. K., and Wei, H. (2019). Droplet digital PCR applications in the tuberculosis world. Tuberculosis 117, 85-92. doi: 10.1016/j.tube.2019.07.001

Pappas, P. G., Lionakis, M. S., Arendrup, M. C., Ostrosky-Zeichner, L., and Kullberg, B. J. (2018). Invasive candidiasis. Nat. Rev. Dis. Primers. 4:18026. doi: $10.1038 /$ nrdp.2018.26

Pfaller, M. A., and Diekema, D. J. (2007). Epidemiology of invasive candidiasis: a persistent public health problem. Clin. Microbiol. Rev. 20, 133-163. doi: 10.1128/CMR.00029-06

Schell, W. A., Benton, J. L., Smith, P. B., Poore, M., Rouse, J. L., Boles, D. J., et al. (2012). Evaluation of a digital microfluidic real-time PCR platform to detect DNA of Candida albicans in blood. Eur. J. Clin. Microbiol. Infect. Dis. 31, 2237-2245. doi: 10.1007/s10096-012-1561-6

Schiavetto, C. M., de Abreu, P. M., von Zeidler, S. V., de Jesus, L. M., Carvalho, R. S., Cirino, M. T., et al. (2021). Human papillomavirus DNA detection by droplet digital PCR in formalin-fixed paraffin-embedded tumor tissue from oropharyngeal squamous cell carcinoma patients. Mol. Diagn. Ther. 25, 59-70. doi: 10.1007/s40291-020-00502-6 
Schroeder, M., Weber, T., Denker, T., Winterland, S., Wichmann, D., Rohde, H., et al. (2020). Epidemiology, clinical characteristics, and outcome of candidemia in critically ill patients in Germany: a single-center retrospective 10-year analysis. Ann. Intensive Care 10:142. doi: 10.1186/s13613-020-00755-8

Sedlak, R. H., Kuypers, J., and Jerome, K. R. (2014). A multiplexed droplet digital PCR assay performs better than qPCR on inhibition prone samples. Diagn. Microbiol. Infect. Dis. 80, 285-286. doi: 10.1016/j.diagmicrobio.2014.09.004

Tulasidas, S., Rao, P., Bhat, S., and Manipura, R. (2018). A study on biofilm production and antifungal drug resistance among Candida species from vulvovaginal and bloodstream infections. Infect Drug Resist. 11, 2443-2448. doi: 10.2147/IDR.S179462

Tumbarello, M., Posteraro, B., Trecarichi, E. M., Fiori, B., Rossi, M., Porta, R., et al. (2007). Biofilm production by Candida species and inadequate antifungal therapy as predictors of mortality for patients with candidemia. J. Clin. Microbiol. 45, 1843-1850. doi: 10.1128/JCM.00131-07

Wisplinghoff, H., Ebbers, J., Geurtz, L., Stefanik, D., Major, Y., Edmond, M. B., et al. (2014). Nosocomial bloodstream infections due to Candida spp. in the USA: species distribution, clinical features and antifungal susceptibilities. Int. J. Antimicrob. Agents 43, 78-81. doi: 10.1016/j.ijantimicag.2013.09.005

Wu, X., Xiao, L., Lin, H., Chen, S., Yang, M., An, W., et al. (2018). Development and application of a droplet digital polymerase chain reaction (ddPCR) for detection and investigation of African swine fever virus. Can. J. Vet. Res. $82,70-74$.
Yang, J., Han, X., Liu, A., Bai, X., Xu, C., Bao, F., et al. (2017). Use of digital droplet PCR to detect mycobacterium tuberculosis DNA in whole blood-derived DNA samples from patients with pulmonary and extrapulmonary tuberculosis. Front. Cell. Infect. Microbiol. 7:369. doi: 10.3389/ fcimb.2017.00369

Conflict of Interest: The authors declare that the research was conducted in the absence of any commercial or financial relationships that could be construed as a potential conflict of interest.

Publisher's Note: All claims expressed in this article are solely those of the authors and do not necessarily represent those of their affiliated organizations, or those of the publisher, the editors and the reviewers. Any product that may be evaluated in this article, or claim that may be made by its manufacturer, is not guaranteed or endorsed by the publisher.

Copyright $\odot 2021$ Chen, Xie, Zhang, Li, Liu, Li, Bian, Jiang, Yang, Li, Feng, Zhang and Shi. This is an open-access article distributed under the terms of the Creative Commons Attribution License (CC BY). The use, distribution or reproduction in other forums is permitted, provided the original author(s) and the copyright owner(s) are credited and that the original publication in this journal is cited, in accordance with accepted academic practice. No use, distribution or reproduction is permitted which does not comply with these terms. 